\title{
EchoGéo
}

$17 \mid 2011$

Activités extractives

\section{Thèmes anciens, approches nouvelles}

Jean-Louis Chaléard

\section{OpenEdition}

Journals

Édition électronique

URL : https://journals.openedition.org/echogeo/12624

DOI : 10.4000/echogeo.12624

ISSN : 1963-1197

\section{Éditeur}

Pôle de recherche pour l'organisation et la diffusion de l'information géographique (CNRS UMR 8586)

Référence électronique

Jean-Louis Chaléard, «Thèmes anciens, approches nouvelles », EchoGéo [En ligne], 17 | 2011, mis en ligne le 27 septembre 2011, consulté le 10 août 2021. URL : http://journals.openedition.org/echogeo/ 12624 ; DOI : https://doi.org/10.4000/echogeo.12624

Ce document a été généré automatiquement le 10 août 2021.

EchoGéo est mis à disposition selon les termes de la licence Creative Commons Attribution - Pas d'Utilisation Commerciale - Pas de Modification 4.0 International (CC BY-NC-ND) 


\title{
Thèmes anciens, approches nouvelles
}

\author{
Jean-Louis Chaléard
}

1 La nouvelle livraison d'Echogéo est largement consacrée aux activités extractives. Ce thème ancien de la géographie humaine (pensons à l'ouvrage de Serge Lerat sur la Géographie des mines, paru en 1971) et au cœur des querelles idéologiques des années 1960-1970, quand les tiers mondistes contestaient l'ordre impérialiste (Le pillage du tiers monde de Pierre Jalée publié en 1968 met au cœur de ses analyses l'exploitation des richesses naturelles des pays du tiers monde par les pays capitalistes développés) est ici revisité par un groupe d'auteurs, souvent jeunes, qui dressent un panorama des enjeux et des nouvelles tendances d'une géographie fortement marquée par les questions politiques et de développement.

2 La hausse continue du prix de l'or depuis quelques années, dans un contexte d'incertitude économique mondiale et de baisse des cours des grandes bourses, comme la découverte toute récente de gisements de pétrole au large de la Guyane française, qu'on nous annonce prometteurs, suffiraient à montrer que le thème fait toujours la une de l'actualité. Il avait déjà été abordé, dans un cadre plus réduit, celui des mines en Afrique de l'ouest, dans un précédent numéro d'Échogéo ( $n^{\circ} 8$, mars-mai 2009). Il s'agit ici d'aller au-delà.

3 Le sujet renvoie à plusieurs questions du monde contemporain. Dans le domaine des activités minières, comme dans beaucoup de secteurs de l'économie mondiale actuelle, on assiste à une redistribution des cartes avec l'arrivée de nouveaux venus sur la scène, que ce soit des petites (tout étant relatif) sociétés d'extraction minière, les juniors, à côté des majors qui ont dominé et dominent encore largement des secteurs entiers de l'exploitation des ressources naturelles (comme le pétrole), et surtout avec l'irruption des pays émergents, en train de redessiner la géographie des productions, des échanges et des pouvoirs.

$4 \quad$ Par ailleurs, la question environnementale est de plus en plus présente dans tout ce qui a trait aux ressources de la planète à cause des dégâts provoqués par l'exploitation inconsidérée des gisements et des inquiétudes sur l'avenir de ressources qui ne sont pas 
renouvelables. À l'heure où le développement doit être " durable », on peut s'interroger sur des matières premières dont l'extraction est source de pollutions (comme l'a montré l'exemple de l'explosion de la plate forme Deepwater Horizon le 20 avril 2010 au large de la Louisiane), dont l'utilisation peut provoquer des incidents graves (c'est le cas du nucléaire) et qui contribuerait au réchauffement climatique.

5 Richesses de pays pauvres? On peut également s'interroger sur le thème de la malédiction des matières premières, le Dutch disease, dont la République Démocratique $\mathrm{du}$ Congo, ex-Zaïre, pays fabuleusement riche en ressources naturelles, en proie à la guerre et où les populations ont de la peine à se nourrir, est l'archétype africain. Les réponses sont sans doute plus nuancées qu'une étude superficielle pourrait le laisser penser, et l'enjeu est aussi de s'interroger sur les multiples bifurcations possibles et sur la diversité des cas.

6 Ces interrogations renvoient bien sûr à d'autres, essentielles pour les géographes. La greffe ou l'enclave? Les changements techniques autant que l'évolution des acteurs conduisent à des interrogations renouvelées sur la place des activités minières à l'intérieur des territoires nationaux ou régionaux. La question est celle de la place de l'activité minière dans des territoires nationaux ou des régions de pays pauvres, qu'elle va irradier ou dont elle sera coupée, lorsque les sociétés minières se contentent d'extraire les ressources et de les acheminer vers les ports à destination de l'étranger. Capitaux, hommes, techniques et consommation sont alors étrangers. Quels bénéfices pour le pays exploité?

7 Ces questions et d'autres sont rappelées par Géraud Magrin et Laetitia Perrier-Bruslé dans sa longue introduction au dossier qui fait un point précis de la question et des problématiques afférentes. Les cinq textes qui suivent illustrent des points particuliers, à l'échelle locale ou mondiale. Quatre ressources sont abordées, différentes dans leur localisation, leur commerce, leur utilisation, ce qui permet de cerner la question sous différents aspects : le pétrole bien sûr, la ressource aujourd'hui la plus stratégique, le fer, l'or et les gemmes, dont on oublie souvent l'importance (et qui ont été sinon la cause du moins au cœur de bien des guerres en Afrique ces deux dernières décennies). Les articles généraux (sur la géographie des pierres précieuses et le rôle des pays émergents, sur les enclaves pétrolières) permettent de replacer les questions à l'échelle mondiale tandis que des articles sur des sujets à plus grande échelle analysent en profondeur quelques thèmes (comme l'implantation chinoise en Afrique avec le projet Belinda au Gabon, l'exploitation aurifère en Guyane qui pose la question de la durabilité de la filière, les relations avec l'environnement humain pauvre dans le cas de l'exploitation de l'or au Burkina Faso). Si tous les aspects d'un thème riche et sensible ne sont pas abordés, comme le souligne Géraud Magrin, ces textes permettent d'explorer largement une question de géographie renouvelée dans ses approches et ses méthodes, et profondément transformée dans le contexte de la mondialisation actuelle.

Dans la rubrique Sur l'image, la contribution de Servane Gueben-Venière revisite moins un thème qu'un outil et une façon d'aborder des questions de géographie, en s'intéressant aux cartes mentales, utilisées de longue date, quelque peu mises de côté pendant un temps et qui sont l'objet d'un regain d'intérêt. L'exemple d'une étude sur la gestion du littoral néerlandais est de montrer toute leur utilité dans le champ de la géographie de l'environnement.

9 L'introduction de Mathieu Durand et l'entretien avec Dominique Amiard, Sur le métier, permettent de s'interroger sur les relations entre élus locaux et géographes. Le 
territoire est au cœur des préoccupations des uns et des autres. Sans doute est-ce pour cela que de nombreux scientifiques ont sauté le pas pour s'impliquer dans l'action publique. Mathieu Durand nous rappelle que cette intervention des géographes dans les affaires de la cité, sous des formes variées, est ancienne. Dominique Amiard nous relate l'expérience d'un géographe maire pour lequel la réflexion et l'action se mêlent étroitement au service l'une de l'autre.

La rubrique Sur l'écrit offre non seulement des comptes rendus de livres, mais essaie aussi d'aller plus loin. C'est le cas du regard croisé proposé par Jérôme Lombard sur deux ouvrages, qui se complètent et se répondent en partie, et qui est accompagné d'un entretien avec les auteurs. Les deux ouvrages concernent la Mauritanie. L'un traite du centre (la capitale, Nouakchott), l'autre de marges (sur les Peuls et l'État en Mauritanie), dans un pays où les ressources minières ont joué et jouent encore un grand rôle. Les deux ouvrages proposent aussi des approches différentes, de géographe pour l'un, d'anthropologue pour l'autre, permettant de confronter des regards disciplinaires singuliers. C'est aussi de cette confrontation que naissent de nouvelles idées et de nouvelles approches. 